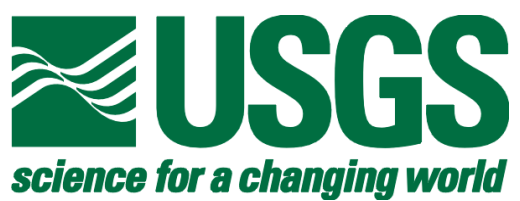

Core Descriptions, Core Photographs, Physical Property Logs and Surface Textural Data of Sediment Cores Recovered from the Continental Shelf of the Monterey Bay National Marine Sanctuary During the Research Cruises M-1-95-MB, P-2-95-MB and P-1-97-MB

By Kevin M. Orzech, Wendy E. Dahl, and Brian D. Edwards

U.S. Geological Survey

345 Middlefield Rd., MS-999

Menlo Park, CA 94025

Open File Report 01-107

2001

This report is preliminary and has not been reviewed for conformity with U.S. Geological Survey editorial standards or with the North American Stratigraphic Code. Any use of trade, product, or firm names is for descriptive purposes only and does not imply endorsements by the U.S. Government. 


\section{Linked Table of Contents}

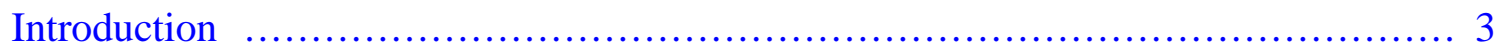

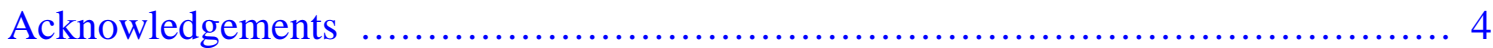

Sample Collection Procedures ...................................................... 4

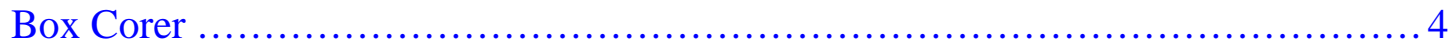

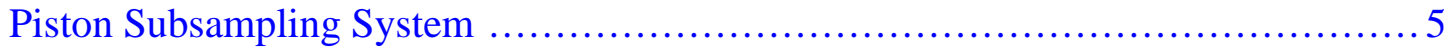

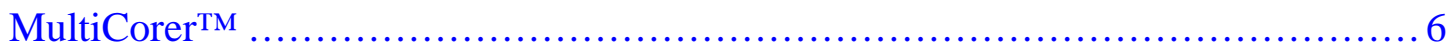

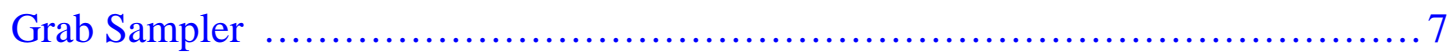

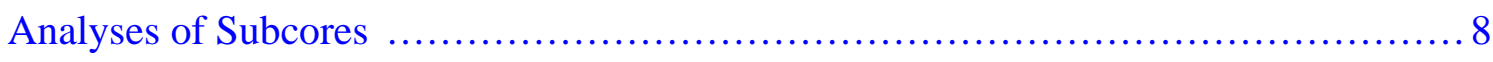

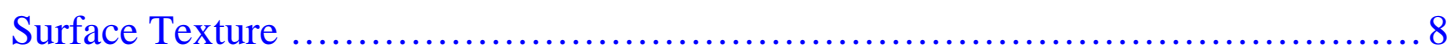

Multi-Sensor Logging of Physical Properties ….................................. 9

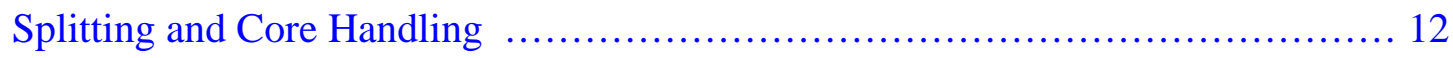

Digital Photography and Image Processing ...................................... 13

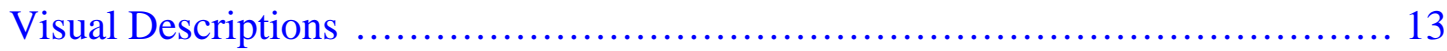

Data Editing and File Management …......................................... 14

Spreadsheet of Station Data f....................................................... 14

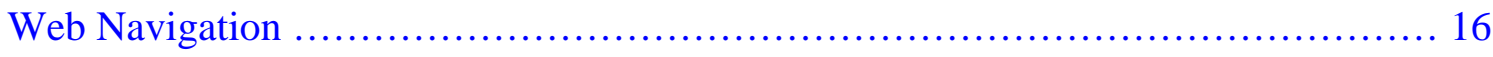

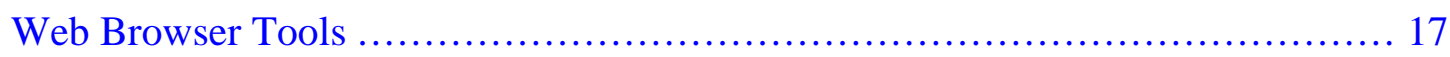

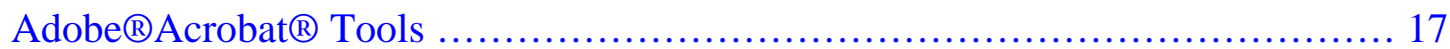

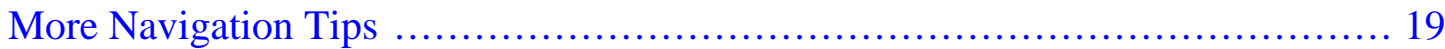

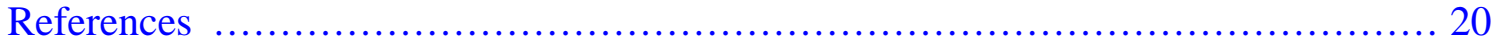

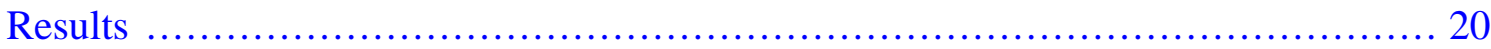

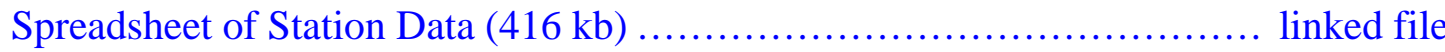

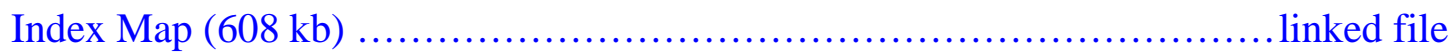

Surface Texture Map (172 kb) ........................................ linked file

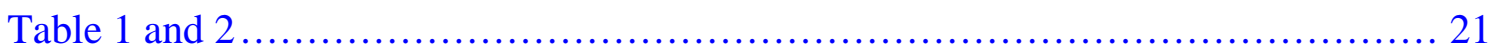

For additional information, contact the corresponding author:

Dr. Brian D. Edwards

U.S. Geological Survey

345 Middlefield Road, MS-999

Menlo Park, CA 94025

650.329.5488 desk

bedwards@usgs.gov 


\section{Introduction}

In response to the 1992 creation of the Monterey Bay National Marine Sanctuary (MBNMS), the United States Geological Survey (USGS) initiated a multiyear investigation of the Sanctuary continental margin. As part of the investigative effort, this report summarizes the shipboard procedures, subsequent laboratory analyses, and data results from three seafloor sampling cruises conducted on the continental shelf between the Monterey peninsula, CA, and San Francisco, CA (fig. 1). The cruises were conducted in 1995 aboard the NOAA Ship McArthur (M-1-95-MB) and in 1995 and 1997 aboard the R/V Point Sur (P-2-95-MB and P-1-97-MB). Scientists and representatives from the Environmental Protection Agency (EPA), the National Oceanic and Atmospheric Administration (NOAA), the California Department of Fish and Game (CDFG), the University of California Santa Cruz (UCSC), and the San Jose State University Moss Landing Marine Laboratory (SJSU-MLML) supported the research effort.

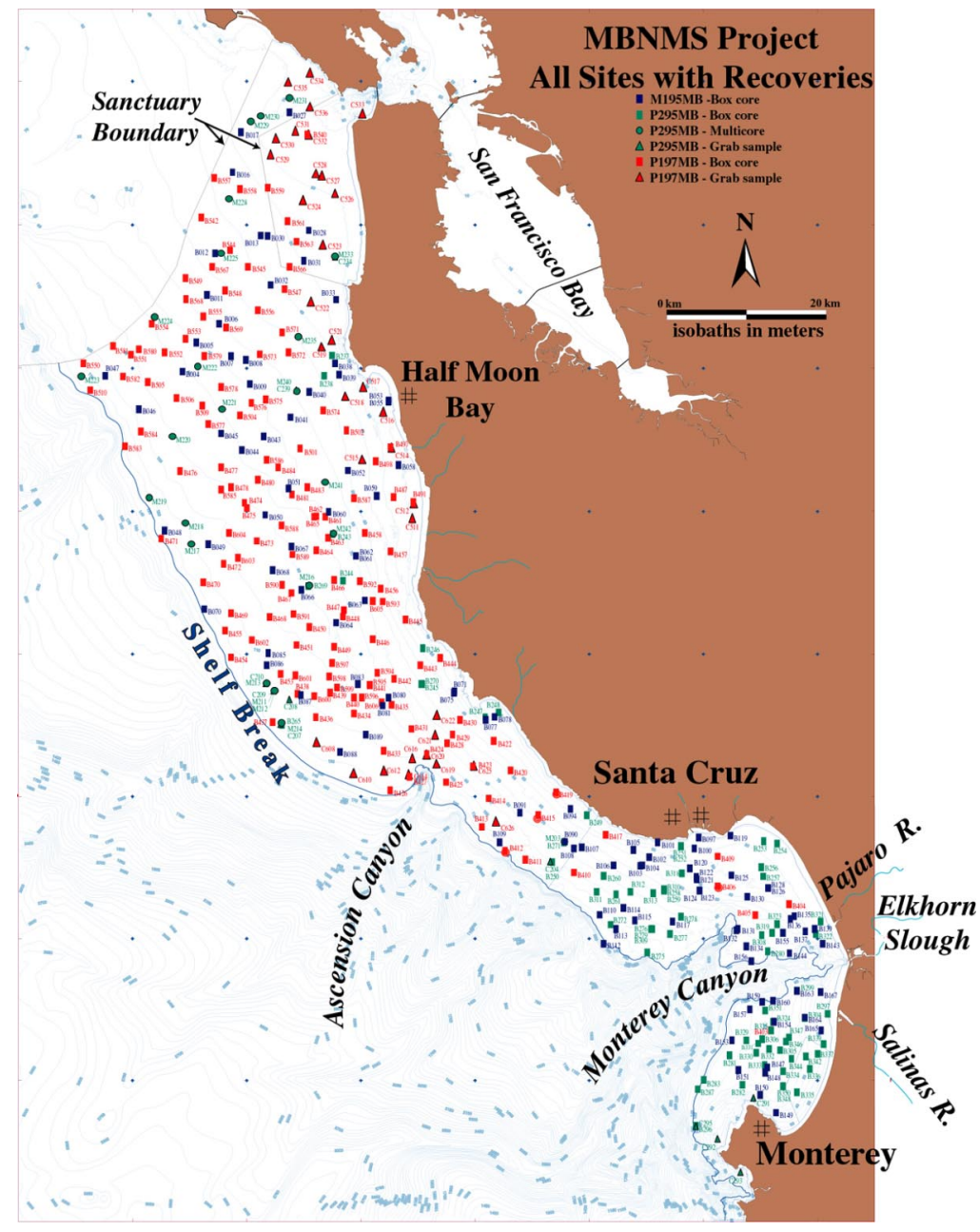

Figure 1. Map showing sample locations and the study region bounded by the shoreline, the shelf break, and the north boundary of the sanctuary.

Three hundred and eighty four stations were occupied during the study. Of these, 342 stations were predetermined based on a horizontally stratified, randomized sampling design following the Environmental Protection Agency EMAP protocol (Overton et al., 1990; Stevens and Olsen, 1991; White et al., 1992). The remaining 42 stations were 
chosen either to sample specific geological targets or to occupy gaps in the randomized design. Ship positioning was determined by differential GPS (accuracy $+/-10 \mathrm{~m}$ ); cores typically are obtained within $50 \mathrm{~m}$ of the predetermined sampling location. Water depth at the time of sampling was determined by use of a $12-\mathrm{kHz}$ acoustic profiler. On the various cruises, either a $1.5-10 \mathrm{kHz}$ Data Sonics Chirp or a $3.5-\mathrm{kHz}$ seismic-reflection profiling system was used to examine and document the local acoustic stratigraphy at each sampling site.

In this report we present sediment descriptions, sediment textural data, physical property logs, station metadata, and photographs of subcores from box cores and MultiCores $^{\mathrm{TM}}$ collected during the cruises. The report is presented in an interactive webbased format accessible with Adobe ${ }^{\circledR}$ Acrobat ${ }^{\circledR}$ downloaded free at this URL:

\section{http://www.adobe.com/prodindex/acrobat/readstep.html}

\section{Acknowledgements}

The authors thank the many scientists, students, and technicians involved in the core collection and sampling program, as well as the capable officers and crews of the NOAA Ship McArthur and the R/V Point Sur (Moss Landing Marine Laboratory, San Jose State University). We also thank H.E. Clifton, J.V. Gardner, and R.L Phillips for the insights they provided with regard to core description and interpretation. D. Ponti created the FileMaker ${ }^{\mathrm{TM}}$ Pro5 database that allowed for easy organization of the core description layouts. M. Medrano and J. Stocking-Albizo performed the textural analyses of the surface sediment. A review by H.J. Lee improved the final version of this Open File Report. This research was funded by the U.S. Geological Survey Monterey Bay National Marine Sanctuary project.

\section{Sample Collection Procedures}

A box corer, a MultiCorer ${ }^{\mathrm{TM}}$ and a grab sampler were used to obtain sediment samples from the continental shelf. Brief descriptions of the three devices and the collection procedures follow. Two tables at the end of this document list the type of subsamples taken from the sediment recovered from each coring device and the scientist in charge of that sampling procedure (link to table 1 and 2).

\section{Box Corer}

Bottom sampling was accomplished primarily by use of the Naval Electronics Laboratory (NEL) - style spade box corer equipped with a removable box (fig. 2). The corer weighs approximately $1500 \mathrm{lbs}$ in air. Immediately prior to sampling, a 35-mm bottom-trip camera system with strobe light photographs the segment of the seafloor beneath the box corer (these photos are available for viewing through the corresponding author). The corer then recovers a nearly undisturbed $0.06 \mathrm{~m}^{2}$ sample $(20 \mathrm{X} 30 \mathrm{~cm})$ up to $60 \mathrm{~cm}$ in length. Early attempts with a larger format $(40-\mathrm{cm} \mathrm{X} \mathrm{40-cm} \mathrm{X} \mathrm{60-cm)} \mathrm{corer}$ were abandoned in favor of the smaller box corer.

Following coring, the corer is returned to the ship's deck, the box is detached and the supernatant seawater is siphoned away from the surface of the sample. The quality of the surface is recorded, and typically a photograph is taken of the core surface. 


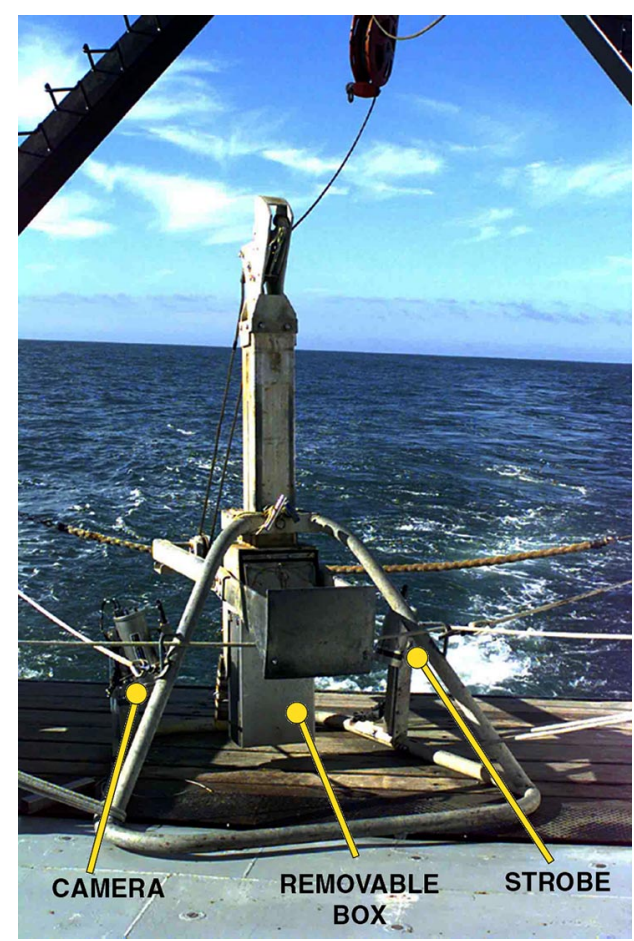

Figure 2. NEL-style Spade Box Corer

\section{Piston Subcoring System and Subsampling.}

The removable box containing the core sample is then placed inside a frame designed to push polybuterate tubes $(8.5-\mathrm{cm}$ diameter $)$ into the sediment using a liner actuator (fig. 3A). The tubes, fitted with a fixed piston, thereby recover a nearly undisturbed subcore of the main sample (fig. 3B).

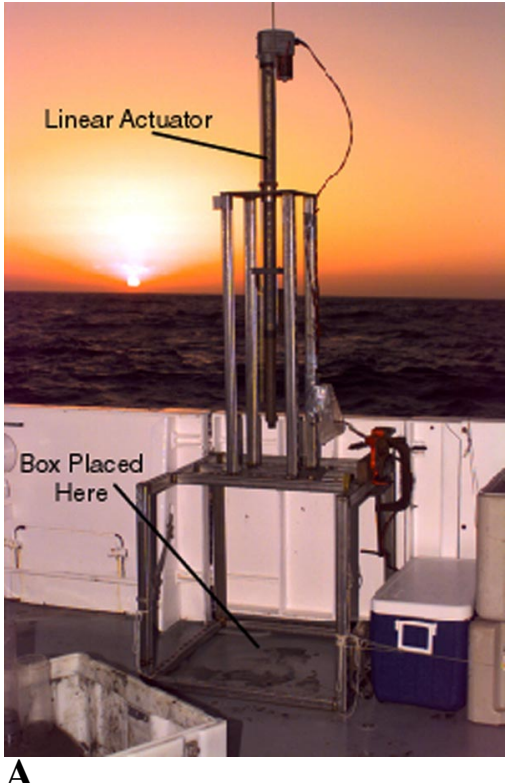

A

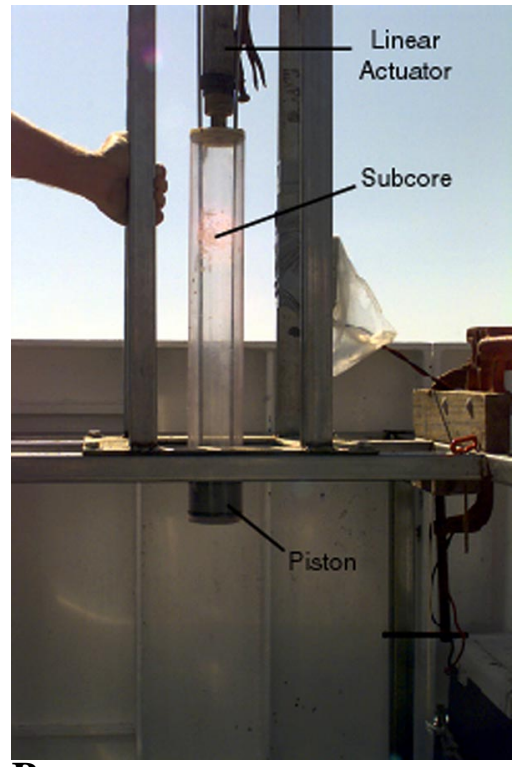

B

Figure 3A and B. USGS-designed piston subcoring system. A) Subcoring frame beneath linear actuator. B) Subcore and piston apparatuses positioned for subsampling. 
Surface samples are then taken (e.g., for texture or microfauna) (fig. 3C), the box faceplate detached, and the subcores removed (fig. 3D). Typically, the remaining bulk sediment is then washed through nested sieves to capture macrofauna.

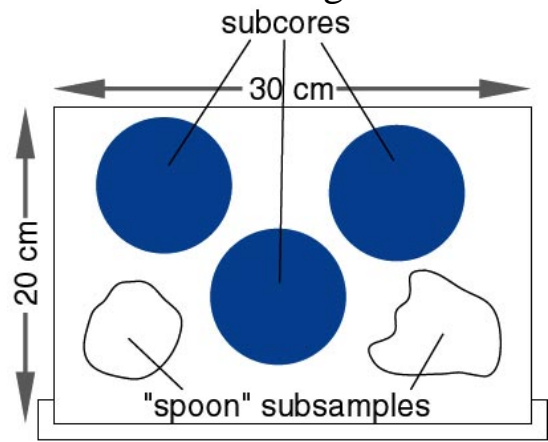

C

Removable Box Faceplate

Figure 3C and D. C) Schematic diagram looking down at the top of the sample showing typical subsampling scheme. D) Deck photograph showing box with subcores inserted, and faceplate removed.

\section{MultiCorer $^{\mathrm{TM}}$}

In addition to the NEL-style Box Corer, a General Oceanics MultiCorer ${ }^{\mathrm{TM}}$ (figs. 4A and 4B) was used during cruise P-2-95-MB aboard the R/V Point Sur.

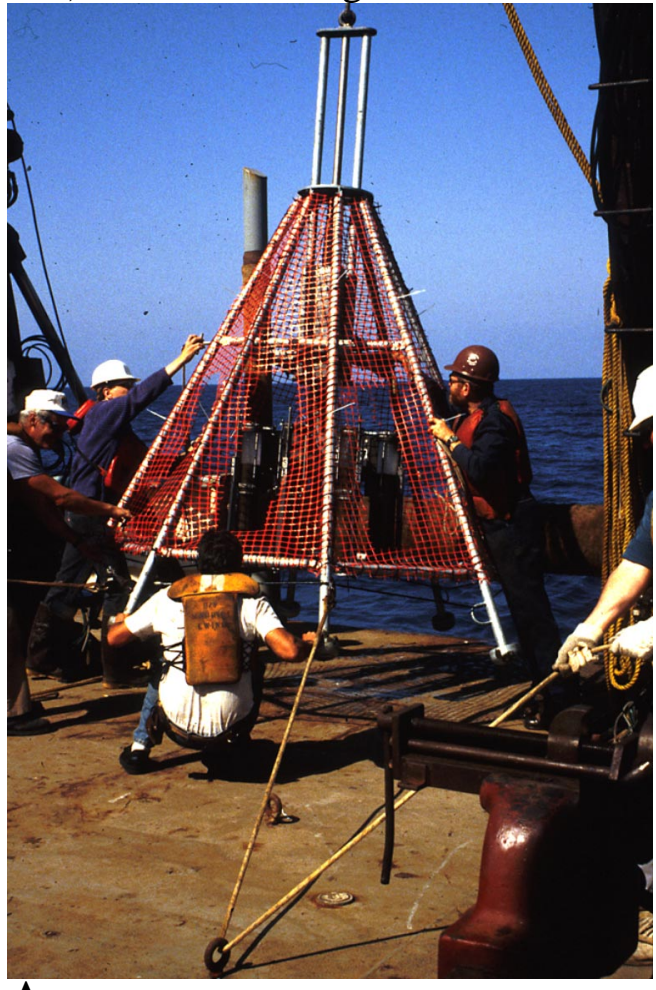

A

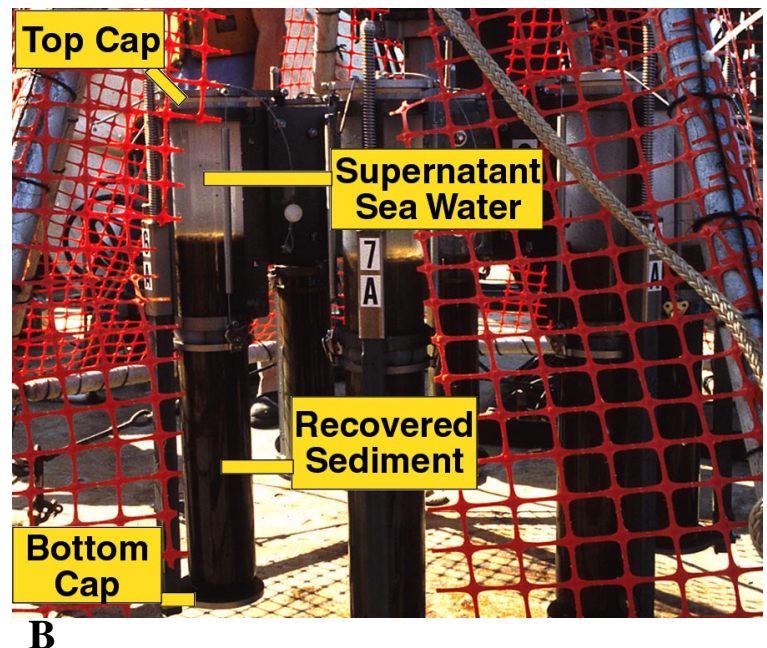

Figure 4A and B. A). General Oceanics MultiCorer ${ }^{\mathrm{TM}}$ being lowered to the deck after recovering samples. B). Detail photograph showing recovered sediment. 
The corer, equipped with cast-acetate tubes, is designed to recover up to eight nearly undisturbed samples simultaneously (fig. 4C). The system is designed to recover a nominal maximum of $45 \mathrm{~cm}$ of sediment in each $10.1-\mathrm{cm}$ diameter and $75-\mathrm{cm}$ long tube thereby allowing $30 \mathrm{~cm}$ of tube volume for supernatant seawater to protect the sediment surface. Each tube is individually sealed with top and bottom caps resulting in an exceptionally high quality sediment-water interface (figs. 4B and 4D).

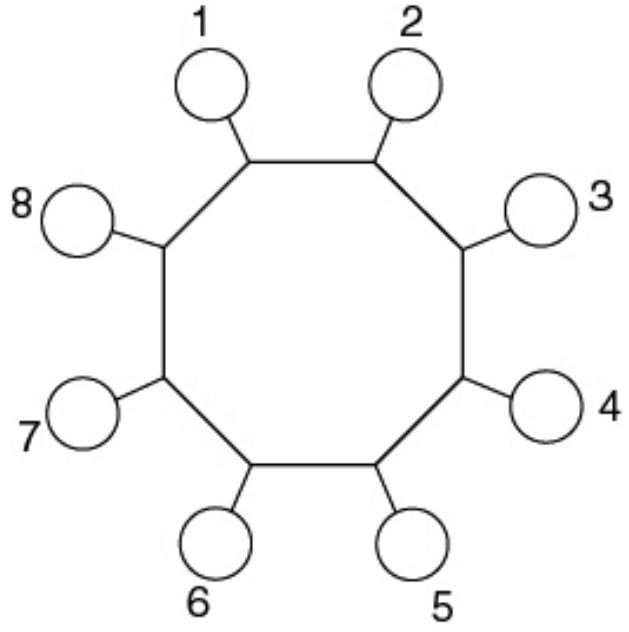

C

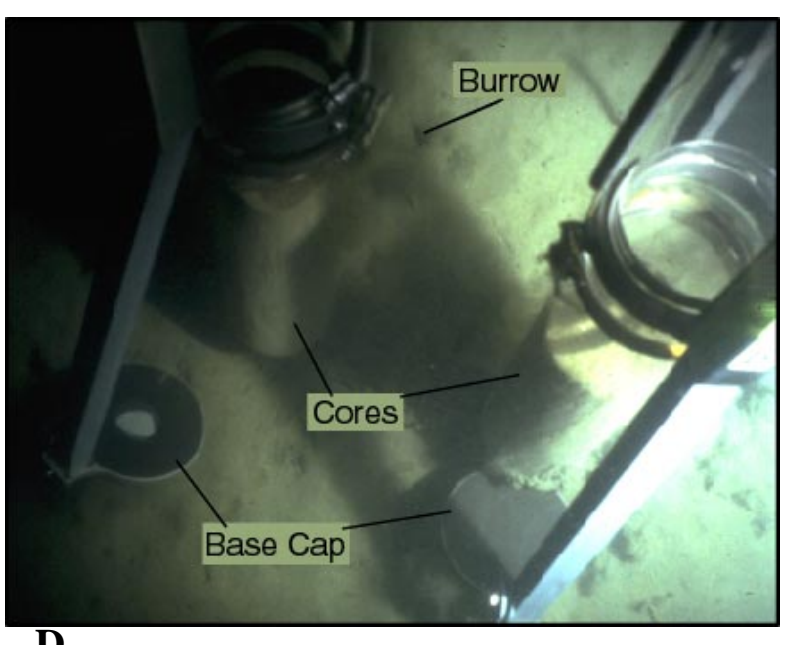

D

Figure 4C and D. C) Schematic of subcores recovered by the MultiCorer ${ }^{\mathrm{TM}}$. D) Bottom photograph taken at the time of sampling. Shows base caps moving to seal the tubes as the corer is being extracted from the seafloor.

Although the MultiCorer ${ }^{\mathrm{TM}}$ recovered samples of excellent quality, operational difficulties in holding position above the corer in shallow water resulted in bending and torquing the corer frame and damaging some of the acetate subcore tubes. Accordingly, we stopped using the MultiCorer ${ }^{\mathrm{TM}}$ midway through the cruise P-2-95-MB.

\section{Grab Sampler}

At sites where high-resolution seismic-reflection geophysics indicated a thinly sedimented, hard, or rocky bottom, a Van Veen-style grab sampler was used to evaluate the bottom type prior to using one of the higher quality (and more fragile and expensive) coring devices (fig. 5). In many cases, these grab samples were the only samples recovered at the site. 

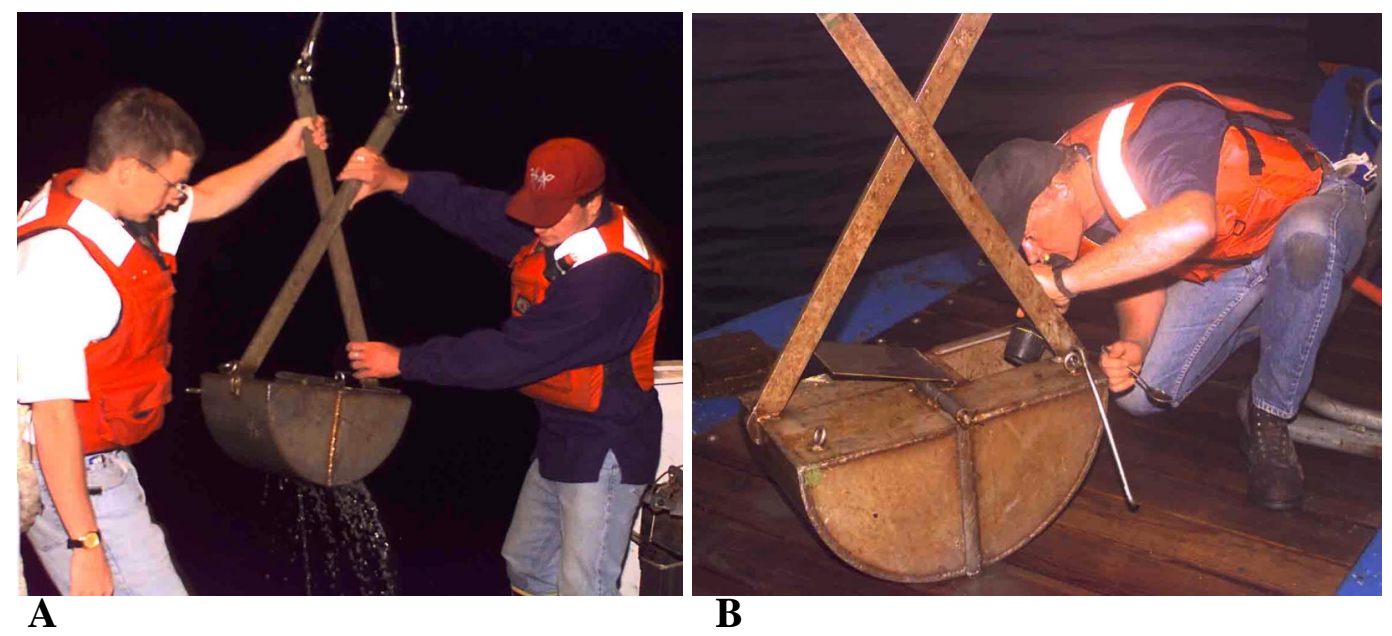

Figure 5. A) Grab sampler being recovered. B) Grab sampler being subsampled.

\section{Analyses of Subcores}

Selected subcores from each box core and MultiCore ${ }^{\mathrm{TM}}$ station were run through a sequence of laboratory procedures that consisted of: 1) non-destructively logging for physical properties, 2) splitting the subcore longitudinally into working and archive halves, 3) photographically documenting the split subcore, 4) describing the visual character and color of the subcore, and 5) taking samples for laboratory analyses. Thirtyone selected subcores were X-rayed to reveal internal physical and/or biogenically produced structures. The X-ray radiographs are not included in this report, but can be viewed by contacting the corresponding author.

\section{Surface Textural Analysis}

Edwards (in press) measured the surface texture at most coring stations from samples taken from the tops of the box cores and MultiCores ${ }^{\mathrm{TM}}$. The results are presented numerically on the Spreadsheet of Station Data $(416 \mathrm{~kb})$ (table 3) and spatially on the Surface Texture Map (172 kb) (fig. 6).

The detailed textural analyses were preformed on the top 1 to $2-\mathrm{cm}$ of recovered sediment. The analyses were done in the lab following a standard grain size multiple step procedure that yields measurements in half phi intervals. Each sample was run through the procedure described below.

First, hydrogen peroxide was added to a sample to remove organic matter. The hydrogen peroxide was then boiled off. Salts were removed by washing the samples with distilled water, centrifuging, and then decanting the water. Next, the sample was segregated into gravel, sand and mud (silt and clay) fractions by washing through standard 1-mm (-1 phi) and 63-micron (3.5 phi) sized sieves. The gravel and sand were dried and weighed. The sand was then run through a USGS-designed rapid sediment analyzer (RSA). The RSA is a $2-\mathrm{m}$-long water-filled tube that generates grain size values in 0.5 phi intervals using the principles of Stokes Law.

The fine fraction $(<62 \mu \mathrm{m})$ was placed into a beaker, disaggregated with sodium hetamexaphosphate (Calgon solution) and left to sit overnight before a representative sample (aliquot) was taken. The aliquot was run through a Micromeretics ${ }^{\mathrm{TM}}$ SediGraph 
which determines grain size of the mud fraction by using a laser that measures the changes in water clarity that correlate with particle settling.

The total amounts within all half-phi-sized increments of gravel, sand and mud were then entered into a USGS-developed statistical program called SedSize. SedSize generated weight percent values in half phi intervals. These values are displayed on the Spreadsheet of Station Data $(416 \mathrm{~kb})$ (table 3).

\section{Multi-Sensor Logging of Physical Properties}

Compressional-wave (P-wave) velocity, wet bulk density and magnetic susceptibility of the subcores were measured in shipboard laboratories using the Schultheiss Geotek Multi-Sensor Logger ${ }^{\mathrm{TM}}$ (MSL) system (fig. 7). Measurements were made on-board ship because P-wave velocity and wet bulk density are state properties that typically change with time and storage. The logging system is described in detail in Kayen et al. (1999), Gardner et al. (1995), Kayen (1994) and Cowen et al. (1994). The following description is exerpted from those reports.

The MSL-system consists of a 4-m-long tracking system and three sequential measurement devices: 1) a compressional-wave (P-wave) velocity and core-diameter sensor, 2) a gamma ray attenuation porosity evaluator (GRAPE), and 3) a magneticsusceptibility sensor. The system is controlled by a Macintosh SE/30 driven by acquisition software written as a HyperCard $\odot$ stack (Kayen and Phi, 1997). Whole core sections up to $1.5 \mathrm{~m}$ in length can be logged with the MSL-system.

Movement along the tracking system is run by a computer-controlled stepper motor that advances the core section at a selectable interval set to $1-\mathrm{cm}$ during logging. Each core section was run consecutively through the sensors, starting with the top (sediment surface) section and progressing through to the bottom of the core. Sixteen of the cores were not logged and have no MSL data.

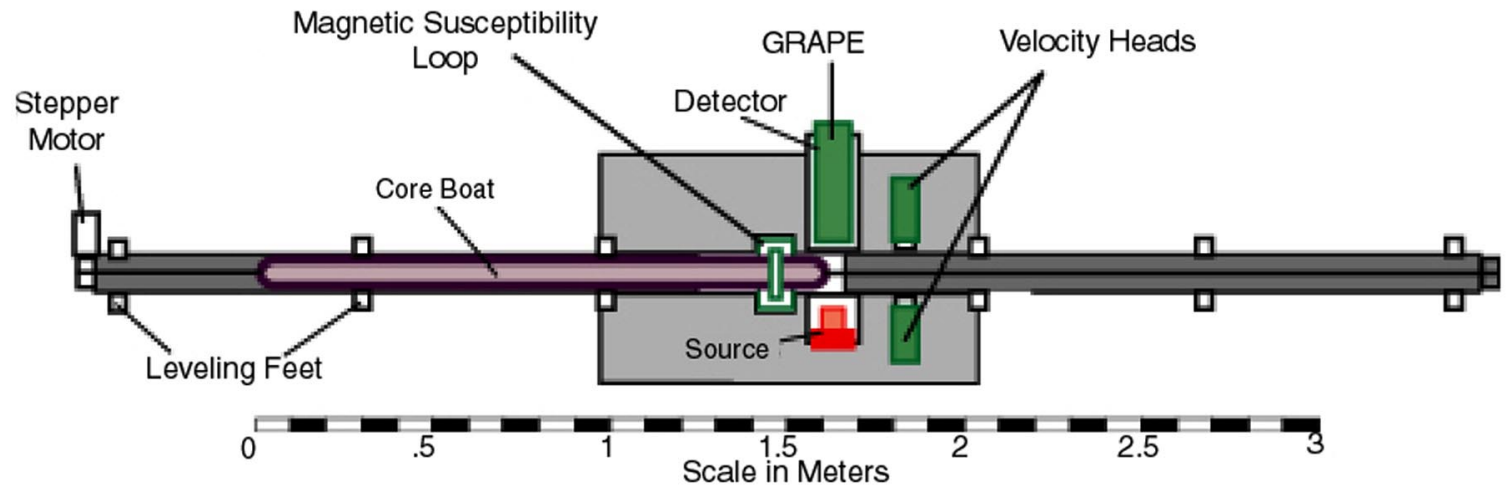

Figure 7A. Plan view of the Multi-Sensor Logger. 


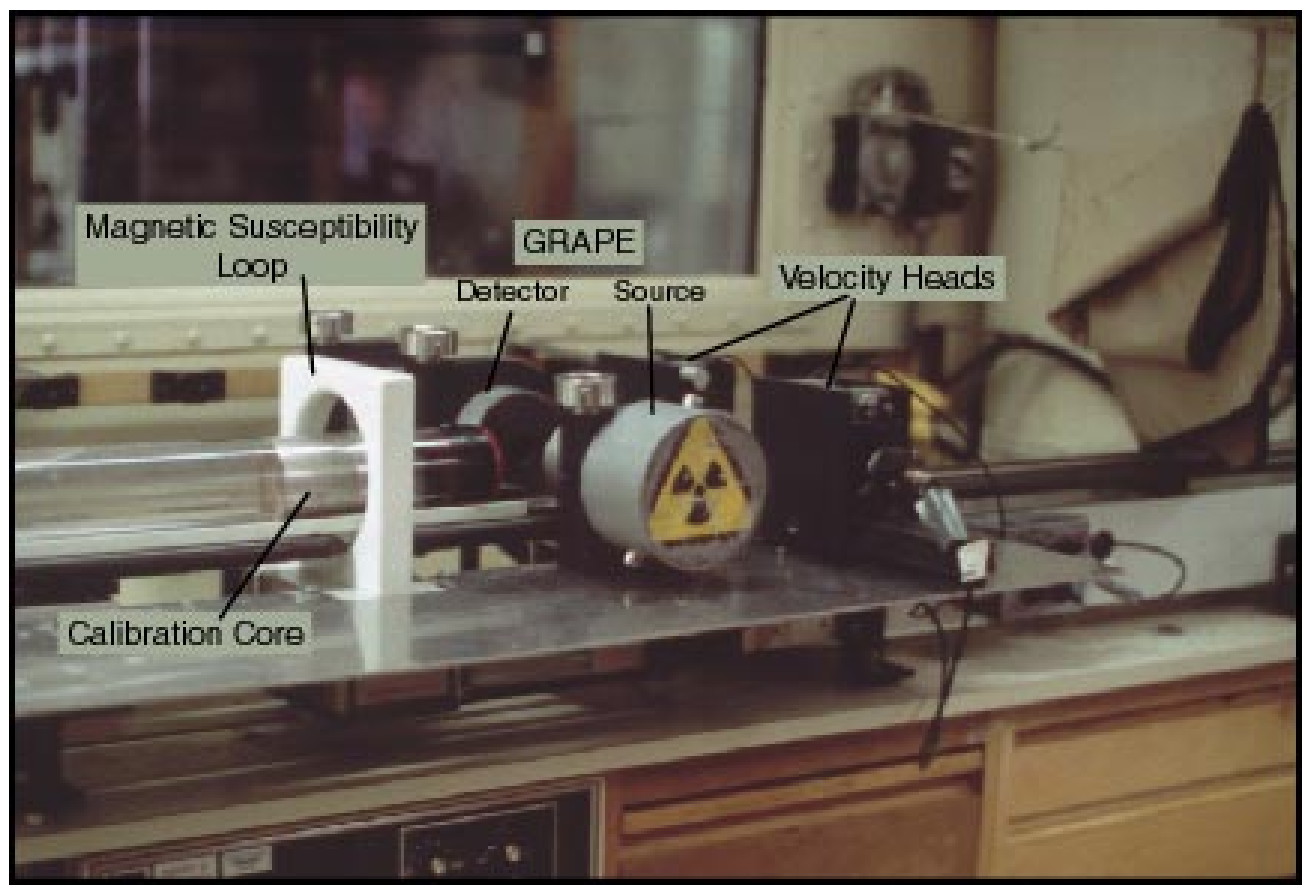

Figure 7B. Photograph of the detectors and sensors at the heart of the MSL system showing the calibration core completing a logging run from right to left.

\section{Compressional-Wave Velocity Measurements}

The compressional-wave velocity sensor is a two-component station. In addition to two sonic transducers, the station incorporates a very accurate $( \pm 0.1 \mathrm{~mm})$ distance measuring sensor (LVDT) that measures the separation of the transducer heads and hence the core diameter. The sensor measures the travel time of a 500-kHz compressional pulse sent through the medial-line of the core. Compressional-wave velocity is sensitive to temperature, so sediment temperature was measured just prior to and following the logging of each core section.

Compressional-wave velocity of the sediment is calculated from the measured core diameter and travel time, with corrections for liner thickness, electronic-signal delays, and core-liner travel time. The compressional-wave velocity $(\mathrm{Vp})$ is calculated as:

$$
\mathrm{Vp}=\frac{\mathrm{D}-2 \mathrm{~L}}{\mathrm{~T}-2 \mathrm{~T}_{\text {liner }}-\mathrm{T}_{\text {electronics }}}
$$

where $\mathrm{D}$ is the whole core outer diameter, $\mathrm{L}$ is the liner thickness, $\mathrm{T}$ is the total travel time, $\mathrm{T}_{\text {liner }}$ is the liner travel time, and $\mathrm{T}_{\text {electronics }}$ is the electronic delay within the transducers, wiring, and electronics packages.

\section{Wet Bulk Density Measurements}

The Gamma-Ray Attenuation Porosity Evaluator (GRAPE) sensor uses a ${ }^{137} \mathrm{Cs}$ solid-source capsule (12 milli-curies at delivery) to produce gamma rays at $0.662 \mathrm{MeV}$. The source capsule is housed in a lead shield and the core is exposed to a beam of gamma 
rays emitted through a collimator hole in the lead shield (fig. 8). The collimator hole is about 11-mm in diameter and 52-mm long. A lead-lined box was also placed over the source and detector during logging for additional shielding. Gamma rays were detected and counted by a Harshaw-type 6S6/1.5B NaI (TI) scintillation detector.

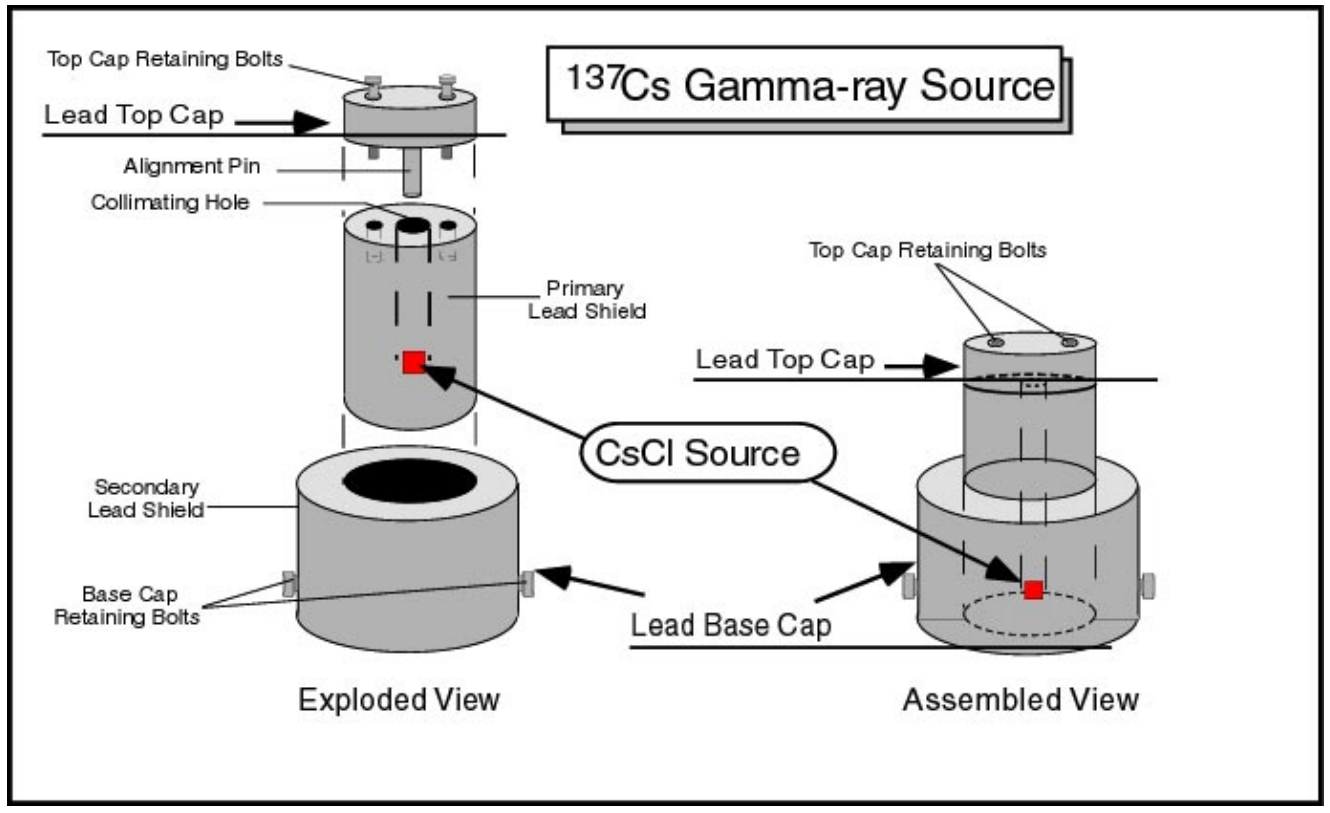

Figure 8. Detached and assembled views showing the construction and lead shielding of the ${ }^{137} \mathrm{Cs}$ source.

The number of gamma rays that pass through the core is detected during a defined time interval. Sediment wet-bulk density can be determined with the following equation, which modifies Lambert's Law to account for the influence of the core liner

$$
\rho_{\mathrm{b}}=\frac{\left\{\ln \mathrm{I}_{\mathrm{O}} / \mathrm{I}-2 \mathrm{~L} * \rho_{\text {liner }} * \mu \text { liner }\right\}}{\mu_{\text {sed }}(\mathrm{D}-2 \mathrm{~L})}
$$

In the formula, $D$ is the outside diameter of the core liner, $L$ is the liner thickness, $\rho_{\text {liner }}$ is the liner density, $\mu_{\text {liner }}$ is the liner Compton scattering coefficient, $\mu_{\text {sed }}$ is the sediment Compton scattering coefficient, I is the number of gamma ray counts that are detected passing through a core during a defined time interval, and $\mathrm{I}_{\mathrm{o}}$ is the number of counts that pass through air during the defined time interval. $\mu_{\text {sed }}$ and $\rho_{\text {liner }} * \mu_{\text {liner }}$ are obtained by logging water and aluminum standards, observing counts, and then backcalculating the needed parameters.

\section{Magnetic Susceptibility Measurements}

The magnetic susceptibility of the sediment is measured directly by a $140 \mathrm{~mm}$ diameter Bartington MS-2 transducer coil. This ring senses the magnetic susceptibility accurately for cores with sediment diameter up to about $91 \mathrm{~mm}$. The box core subcores and MultiCores ${ }^{\mathrm{TM}}$ have inside diameters of about $80 \mathrm{~mm}$ and $95 \mathrm{~mm}$, respectively. Accordingly, every magnetic susceptibility reading made by the MS-2 system was 
corrected using a compensation graph for different diameters given in the Bartington Manual.

All magnetic susceptibility readings are unitless and in the cgs system. Graphed values in this report are listed in whole numbers for convenience. They need to be multiplied by $10^{-6}$ to obtain the correct value. Because the range of magnetic susceptibility values varied enormously throughout the study, three data ranges for the abscissa $\left(0-100,0-250\right.$ and $\left.0-1000 * 10^{-6} \mathrm{cgs}\right)$ were used. The magnetic susceptibility graphs in this report depict the smallest of these ranges that incorporates all magnetic susceptibility data for a core.

\section{Splitting and Core Handling}

After shipboard logging with the MSL system, the polybuterate subcores taken from the box cores were split lengthwise in an on-shore laboratory using a splitter patterned after the Deep Sea Drilling Project design. The splitter uses a capstan and a rope to pull a pair of utility blades down the length of the core (fig. 9). The blades cut only the liner, not the sediment. The blades are mounted in housings on either side of the core. The housings, attached to tracks by a set of bearings, are pulled along the outside of a channel on which the core rests. Thus, the blades simultaneously slice opposing sides of the core liner.

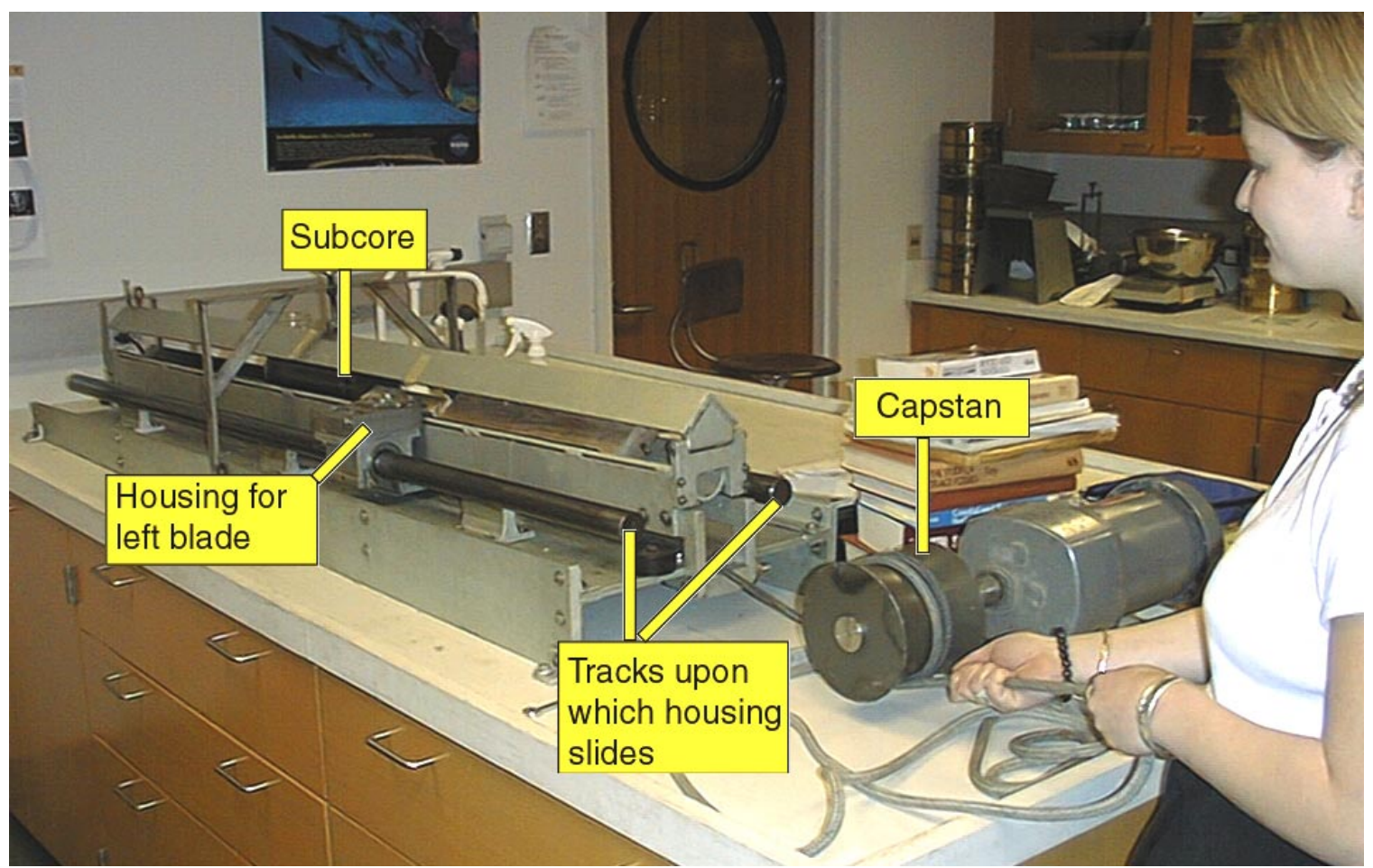

Figure 9. Finishing the splitting of a core liner with the splitter.

The core splitter was ineffective in cutting the hard, cast acetate MultiCore ${ }^{\mathrm{TM}}$ liners . Accordingly, these liners were cut longitudinally by use of a table saw. After either type of liner was cut, a tensioned wire was pulled through the sediment from top to bottom to yield two equal sized half cores - an archive and a working half (fig. 10). 


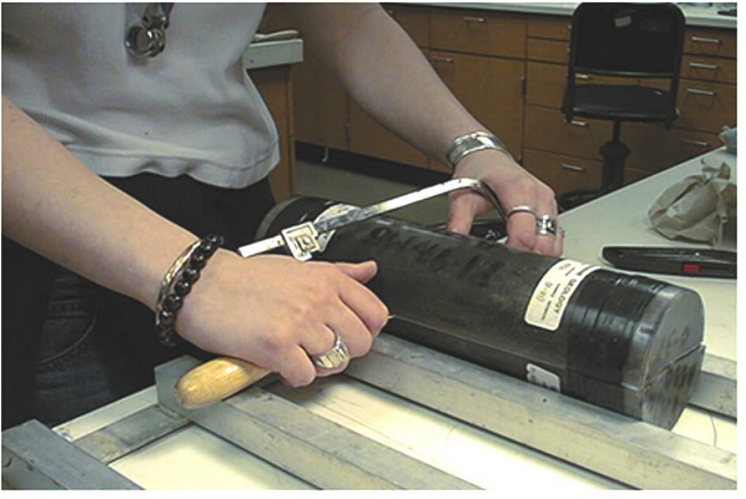

A

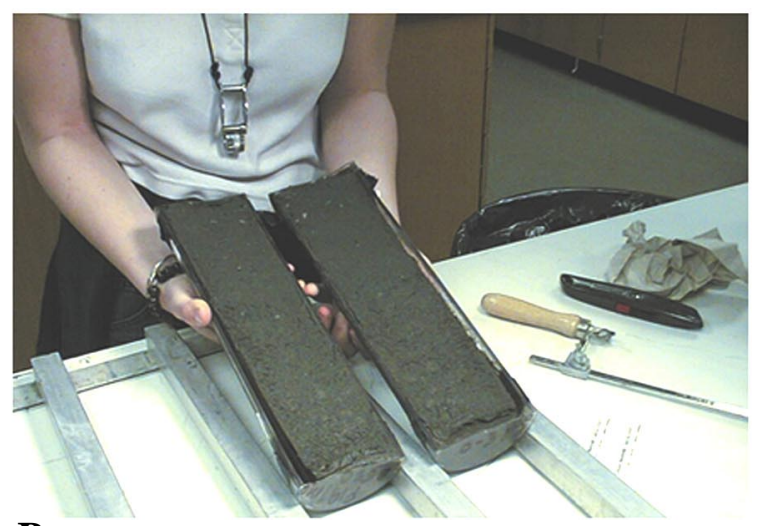

B

Figure 10. A) Pulling a tensioned wire through a subcore. B) Exposed surfaces of working and archive halves of a subcore.

\section{Digital Photography and Image Processing}

Archive halves were placed on a core rack adjacent to a ruler and a Munsell Soil Color Chart. The rack was positioned on a photo stand under a Kodak ${ }^{\mathrm{TM}}$ Professional DCS 620 digital camera with a Nikon ${ }^{\mathrm{TM}} 28-\mathrm{mm}$ apochromatic lens. All images were imported into Adobe ${ }^{\circledR} P h o t o s h o p \circledR ~ 5.5$. Images were cropped to 30-cm increments in Adobe ${ }^{\circledR}$ Photoshop ${ }^{\circledR}$. For cores longer than $30-\mathrm{cm}$, a 0 to 30 and a 30 to $60-\mathrm{cm}$ image were meshed to yield a composite image of the whole core. These images contain the color charts and ruler so that they may be manipulated to obtain the true color of the sediment (the composite images are not included as part of this report but may be accessed by contacting the corresponding author). Next, the composite image was cropped to an image of the core sediment only. Brightness, sharpness and color balance adjustments were made to this image with two primary goals: to obtain an accurate color of the sediment and to enhance the visual features of the sediment. These final images are generally slightly lighter colored than the actual core sediment. Last, the images were placed into a FileMaker ${ }^{\mathrm{TM}}$ Pro5 database and are presented as part of this report.

\section{Visual Descriptions}

Visual descriptions were performed immediately after photographing the core using the working and archive halves side-by-side. Descriptions consisted of grain size, sorting, color, macro- and ichnofossil content, bioturbation intensity, lithologic accessories and physical structures. A grain size estimate was determined for at least every $10-\mathrm{cm}$ core interval by obtaining a small sample from the working half and using a hand lens with a grain size card that is delineated in half phi intervals. A simple visual sorting of the sediments was obtained by viewing the same sample with the lens and card. This visual sorting and grain size are not to be confused with the detailed surface textural data described above. Visual sorting was not determined for mud-rich sediments or for many of the first cores described (mostly the P-2-95-MB cruise). Sediment color was determined by placing Munsell Soil Color Charts over the sediment and finding the best match. Bioturbation intensity was assessed visually both after splitting and when the sediment surface was smoothed with a metal spatula. The degree of bioturbation was catalogued under one of the following 5 categories: Abundant $(>60 \%$ of the sediment disturbed by bioturbation), Common (30-60\%), Moderate (10-30\%), Rare $(<10 \%)$, and None Observed. Physical structures, macro- and ichnofossils, and lithologic accessories were also listed from visual assessment of the cores. X-ray radiographs for 31 cores provided more details of structures and bioturbation. 
After the cores were described, both halves were sealed with Handi-Wrap®, placed in plastic D-tubes and stored in a refrigerator that maintained temperatures between $34^{\circ}$ and $40^{\circ} \mathrm{F}$. The reefer is at the USGS in Menlo Park, CA.

\section{Data Editing and File Management}

Raw MSL data for sixteen cores were recalculated because of poor calibration at the time of logging. All MSL data was further editted by the following rules:

1) Discarded values fell outside of the range 1.3-2.2 g/cc for wet bulk density and 1.4$1.8 \mathrm{~km} / \mathrm{sec}$ for P-wave velocity. No magnetic susceptibility values were discarded.

2) If a density value was edited out, the velocity and magnetic susceptibility values of the same increment were also edited out regardless of their value.

3) When the last density, velocity or magnetics value of the core was abnormally low compared with the rest of the MSL data for the core, the datum was edited out. Values successively up-core from this bad datum were edited out following the same procedure. Data was removed only from the lower 1 to $4 \mathrm{~cm}$ of core. These values were considered inaccurate and usually low because poorly consolidated sediment or less sediment occupied the ends of cores.

The visual descriptions and editted MSL data were entered into the AppleCORE ${ }^{\mathrm{TM}}$ 8.1 a program - a customizable core graphing program. During the process, this visual data was cross-checked for errors by comparing the digital photograph of the core with the data and visually reassessing any cores with questionable data. Next, core photographs and AppleCORE ${ }^{\mathrm{TM}}$ layouts were imported into a FileMaker ${ }^{\mathrm{TM}}$ Pro5 database designed by the USGS. Within the database, the final core layouts were assembled on one sheet by combining the AppleCORETM layouts, photos, a border and the proper headings of core and cruise I.D., latitude and longitude, water depth, and date sampled. Each of these files was saved as an Adobe®Acrobat ${ }^{\circledR}$ PDF file and linked to the Index Map (608 kb) (fig. 11), Legend (fig. 12a and 12b) and Spreadsheet of Station Data $(416 \mathrm{~kb})$ (table 3) using Adobe®Acrobat ${ }^{\circledR} 4.05$.

\section{Spreadsheet of Station Data}

The Spreadsheet of Station Data (416 kb) (table 3) catalogues all location, date, time, recovery, and sampling and surface textural data for each core station. An Adobe ${ }^{\circledR}$ Acrobat ${ }^{\circledR} 4.0$ version of the spreadsheet contains links to each core description and photo sheet. To access the links click on the blue Station ID (core ID) numbers. Use the back button to return to the spreadsheet. A Microsoft@Excel 98 version of the same spreadsheet is included in the report folder and linked to the Home Page so that the data may be resorted and organized for the viewer's needs. Use the spreadsheet headings key below to understanding the column headings of the Spreadsheet of Station Data $(416 \mathrm{~kb})$ (table 3). The heading bar on the spreadsheet links to this key:

CRUISE ID: Represents the cruise identification number for the sample station.

STATION ID: Sample station identification number, consistent with the linked core identification number on the Index Map (608 kb) (fig. 11). The first letter of the station number represents the type of sampling device used at the station (B-Box corer, C - Grab sampler, and M - MultiCorer ${ }^{\mathrm{TM}}$ ).

JD/TIME (GMT): Time and date of sampling expressed in Julian Day (JD)/Greenwich Mean Time (GMT). The Julian day is expressed beginning with January $1^{\text {st }}$ as 
day 1 and December $31^{\text {st }}$ as day 365 (366 during leap year). Greenwich Mean Time is the time at Greenwich, England located at 0.00 degrees longitude.

$\underline{D E P T H}(m):$ Depth in meters at the sample location.

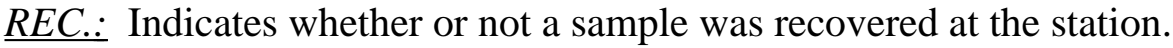

LAT. and LONG. DD: Latitude and longitude of the station location in decimal degrees.

SURF. QUAL.: Indicates a sediment surface quality value that was recorded once the box core was returned to the ship. Values are from 0 to 5 , with 0 being the lowest value of quality. "No" shows that no value was recorded for the station.

REC. $(\mathrm{cm}):$ The length of the sediment sample recovered at the station. For box cores, this represents the height of the sediment in the box cores prior to subsampling.

$\underline{S S}$ : Indicates if a subcore was taken for stratigraphy and sedimentation studies.

SS $(\mathrm{cm}):$ The length of the SS subcore. Occasionally the length of the SS subcore was different than the total recovery due to an uneven surface of the box core sediment or air in the core liners.

SURF.TX.: Indicates whether a sample was taken for surface texture analyses.

MF SURF.: Indicates whether a surface sample was taken for microfauna studies.

MF CORE, GT, and GC: Indicates if a subcore was taken for microfauna, geotechnical, or geochemical studies, respectively.

TMSO: Indicates if a subcore was taken for chemical study of metals, pesticides, and PCBs.

MB: Indicates if macrobenthos subcores were taken. The table lists four MB columns because up to four MB subcores were taken at individual stations.

MB WASH: Indicates if sediment in the box core was washed and sampled for macrobenthos studies.

MICRO. ORG.: Indicates if a 5-cm subcore sample was taken for micro-organism studies.

$\underline{P B}$ : Indicates if a subcore was taken for lead-210 dating purposes.

BULK, OTHER SAMPLE: Indicates if bulk or other samples or subcores were taken.

CORE DES. AND PHOTO: Indicates if core descriptions and core photographs are available and linked to the index map of this report.

BOTTOM PHOTO: Indicates if a bottom photograph was taken at the sample station.

MSL DATA: Indicates if multi-sensor logger data were taken and are linked to the station identification number on the index map. 
C14 SAMPLES and DIATOM SAMPLES: Indicates which cores were sub-sampled for carbon-14 radiometric dating and diatom analyses.

GRAIN SIZE DATA: Indicates if detailed surface textural data was measured in the lab and listed in the columns to the right of this column.

\# OF SIZE CLASSES: Indicates the number of 0.5 phi-size classes represented in each surface textural sample.

COARSE SIZE LIMIT: Represents the coarsest phi-size grains of the surface textural sample.

TOTAL WEIGHT OF SAMPLE $(g)$ : Shows the total weight in grams of the entire sample used for grain size analysis.

$-1.0,-0.5, \ldots 14.00:$ Shows the weight percents for each phi-size of the textural sample.

TOTAL 100\%: Indicates the sum total of the phi-size weight percents (which ideally is $100 \%)$.

\% GRAVEL, SAND, SILT, CLAY and MUD: Represents the percentages of gravel, sand, silt, clay, and mud in each sample.

GRAVEL/SAND, SAND/SILT, SILT/CLAY, SAND/CLAY, SAND/MUD, and GRAVEL/MUD: Shows the ratios of the various size classes.

$\underline{1^{S T} M O M E N T(m e a n):}$ Indicates the mean grain size of each sample.

VARIANCE: Shows a value representation of dispersion calculated as the sum of the squared deviations from the mean grain size for each phi-size class divided by the number of phi-size classes with data.

STANDARD DEVIATION: Indicates the deviation from the mean. It is a value measure of dispersion calculated as the square root of the variance.

每D MOMENT: Measure of skewness - the asymmetry of the distribution (Carver, 1971).

$4^{T H}$ MOMENT: Measure of kurtosis - how "humped" or "peaked" the central part of the distribution appears (Carver, 1971).

COMMENTS: Provides any additional information for each sample station.

\section{Web Navigation}

On most computers, the open file report will open in the Adobe ${ }^{\circledR}$ Acrobat ${ }^{\circledR} 4.0$ program within your Internet browser. (It fails to do this when an Adobe $₫$ Acrobat ${ }^{\circledR} 4.0$ plug-in is not installed within the computer preferences. Installing the free plug-in (available at http://www.adobe.com/prodindex/acrobat/readstep.html) or downloading the entire report onto your desktop will mitigate this problem). The Adobe $₫$ Acrobat $\AA$ software and Internet browser tools will enable users to view all images and to link from the Index Map (608 kb) (fig. 11) or Spreadsheet of Station Data (416 kb) (table 3) to each core description sheet, the Surface Texture Map (172 kb) (fig. 6), the Home Page and the linked Text and Table of Contents of this text document. Use and understanding of three 
main browser tool buttons (Back, Forward and Reload (Netscape Navigator ${ }^{\mathrm{TM}}$ ) or Refresh (Internet Explorer ${ }^{\mathrm{TM}}$ )) and six main Adobe ${ }^{\circledR A}$ Acrobat ${ }^{\circledR}$ buttons (the Hand Tool, Zoom Tool, Go to Previous View, Go to Next View, Fit in View, and Find) will enable you to navigate smoothly through the report.

\section{Web Browser Tools}

The Back and Forward buttons on your browser tool bar (fig. 13) will take you to and from files that have been previously opened. Some browsers have run into the problem of large files that fail to load or appear when a link is pressed. When this happens without the appearance of an explanation window use the Reload button (fig. 13) in Netscape Navigator ${ }^{\mathrm{TM}}$ and the Refresh button in Internet Explorer ${ }^{\mathrm{TM}}$. The failure of files to load occurs more commonly on computers with small memory particularly when loading the Index Map.

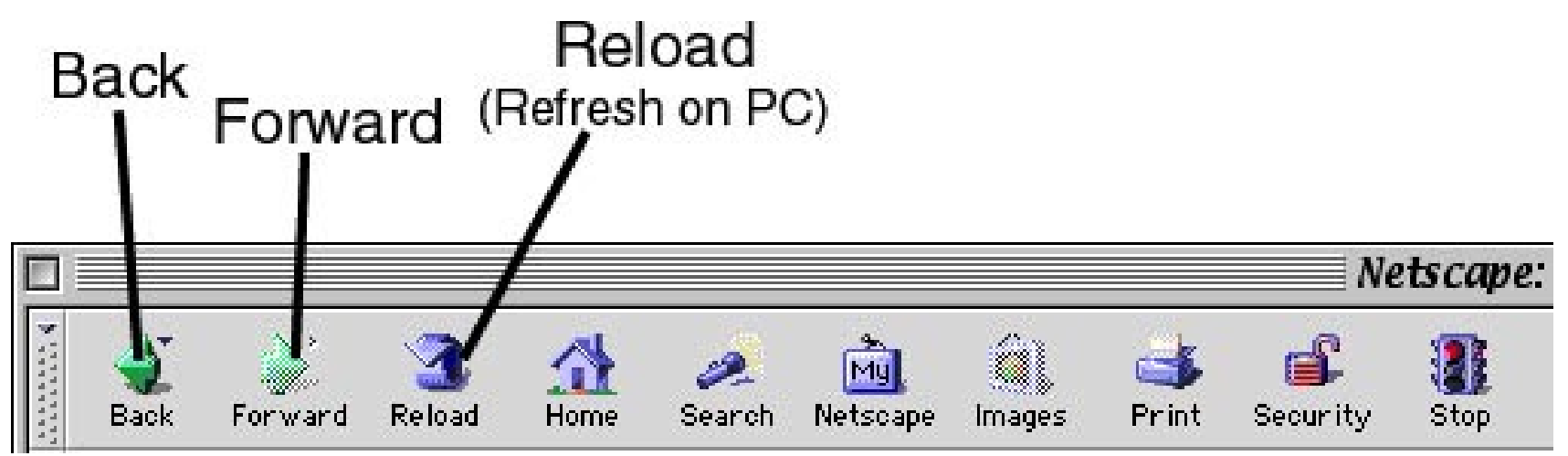

Figure 13. Right side of the Netscape Navigator ${ }^{\mathrm{TM}} 4.08$ tool bar.

\section{Adobe ${ }^{\circledR A c r o b a t} \AA 4.0$ Tools}

Use the Adobe®Acrobat ${ }^{\circledR}$ tools (fig. 14) to access the links, to zoom in and out of a page, to change the view size, to navigate within this text document, and to find key words or numbers on a particular view. To navigate between the linked pages click on the Hand Tool on the tool bar (fig. 14 and 15). The cursor will change to a hand. Once placed over a link, the hand changes to a hand with a pointing finger. Clicking on the link at that time brings up the linked file.

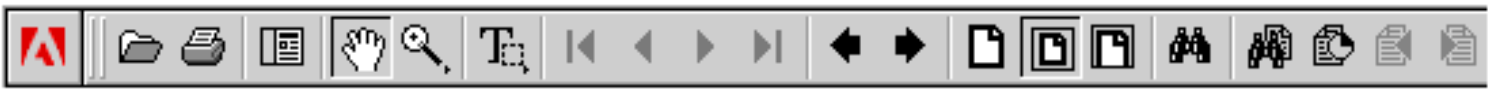

Figure 14. Adobe ${ }^{\circledR}$ Acrobat ${ }^{\circledR}$ Reader4.0 tool bar. 


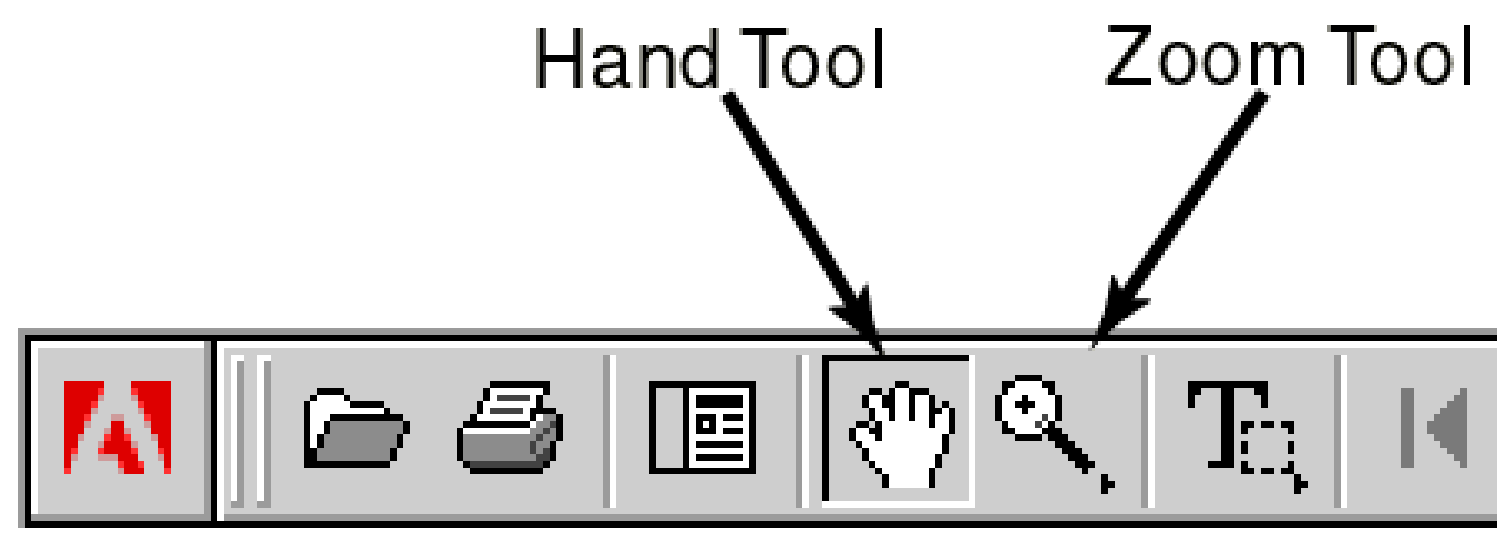

Figure 15. Left side of Adobe®Acrobat $\AA$ Reader4.0 tool bar.

You may zoom in and out of the core logs and map using the Zoom Tool on the tool bar (fig. 15) or by pressing the Macintosh ${ }^{\mathrm{TM}}$ keys command-minus, -plus or -zero to zoom in, out and to a full screen view respectively. Clicking on the Fit in Window icon (fig. 16) on the tool bar also yields a full screen view.

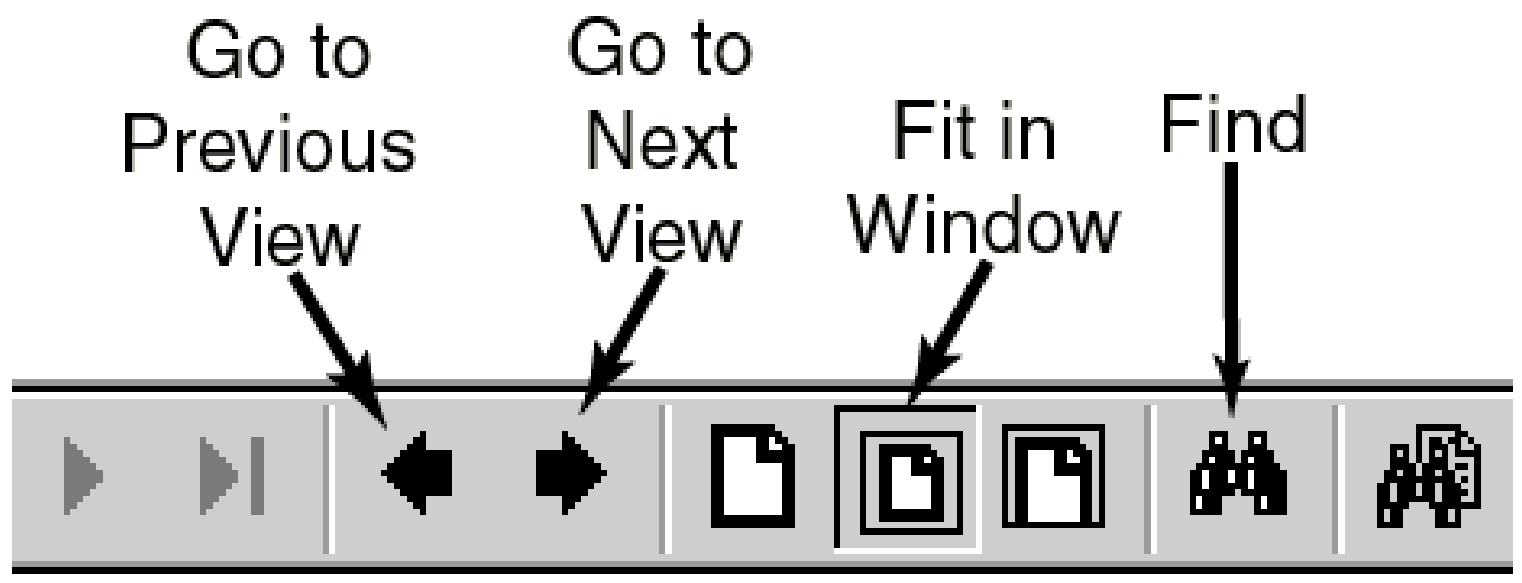

Figure 16. Middle of Adobe $\AA A$ crobat $\AA$ Reader4.0 tool bar.

Brief directions for the important Adobe ${ }^{\circledR}$ Acrobat ${ }^{\circledR} 4.0$ tools follows:

Hand Tool: The tool enables linking from one file to another. Place the hand over a link and click the mouse to access the linked file. (Also the hand alone may be used to move the entire document on the page).

The keyboard command to access the hand is the space bar.

Zoom Tool: Use this icon to zoom in and out on a view. Click the mouse to zoom in.

Alternatively, trace a rectangle around a region by holding down the left mouse button while tracing, and release the button to zoom in.

The Macintosh ${ }^{\mathrm{TM}}$ keyboard command for zooming out is the option key.

The Macintosh ${ }^{\mathrm{TM}}$ keyboard command for accessing the zoom tool is command/apple + space bar.

Go to Previous View and Go to Next View (Back and Forward arrow buttons): To scroll forward and backward through the most recent screens that you have viewed, use the forward and backward arrows (fig. 16). These views include different zoom 
views of the same file. Use these buttons repeatedly if you wish to get back to a screen that you have recently viewed that does not have a direct link from the page that you are on, such as going from the linked spreadsheet to core layout and back to the spreadsheet.

Fit in Window: The command resizes the current image to fit fully onto the screen.

Find: By opening up a text window, this command will find the text you type if it exists on the current file that is open. Use this command on the index map or spreadsheet to locate a particular core by I.D. number or to locate other text data. The Macintosh ${ }^{\mathrm{TM}}$ keyboard command is command/apple $+F$.

\section{More Navigation Tips}

- Use Adobe®Acrobat ${ }^{\circledR}$ 's Zoom Tool and Hand Tool and the browser's Back button to perform most navigation throughout the site; linking from the index map to the core layouts works most effectively.

- Click on the Core I.D. text, not the colored square, on the index map using the Hand $\underline{\text { Tool }}$ to access links to the core layouts (fig. 17).

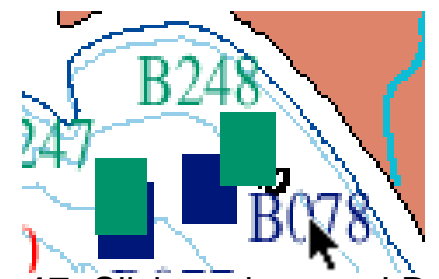

Figure 17. Click on the core I.D. text, not the colored square, to access the linked file.

- Bookmarks in a column at the right side of the Index Map enables users to quickly zoom to the main regions of the Index Map $(608 \mathrm{~kb})$ (fig. 11). If your browser is not set up for this when the map is open, click on "Window - Show Bookmarks" and ensure that "Hide After Use" is not checked within the bookmark submenu.

- Working with spreadsheet data: The Microsoft@Excel spreadsheet contains the same data as the Adobe $®$ Acrobat ${ }^{\circledR}$ spreadsheet. It may be manipulated and sorted to refine your search. On the Microsoft ${ }$ Excel spreadsheet, sort the sample data by using "Sort" under "Data" on the header bar. You may sort the data by any of the column headings. For example, you may want to view all cores from water depths of 50 to $100 \mathrm{~m}$. Highlight all data below all of the column headings; then use Data-Sort and choose to "sort by" the Depth column. With this list either open the fixed Adobe ${ }^{\circ}$ Acrobat ${ }^{\circledR}$ spreadsheet or the index map. Use the Find Tool to locate the cores of interest and click on the link to access the core layouts.

- To view many core layouts at once open the files individually from the main report file folder. Web browsers and Adobe ${ }^{\circledR}$ Acrobat ${ }^{\circledR}$ allow viewers to toggle back to the open files under the Go and Window menus respectively. To maneuver through all the screens that have been viewed (including zoomed views) since opening the program, use the back and forward arrows on the browser tool bar (fig. 16).

- Five percent of the cores sites on the index map have no box or MultiCore ${ }^{\mathrm{TM}}$ recovery. The links from these sites will specify the type of sample recovered or reasons for failure to obtain a full core. 


\section{References}

Carver, R.E., 1971, Procedures in Sedimentary Petrology: John Wiley and Sons, Inc., New York, $653 \mathrm{p}$.

Cowen, E.A., Powell, R.D., Carlson, P.R., Kayen, R.E., Cai, J., Seramur, K.C., and Zellers, S.D., 1994, Cruise Report: R/V ALPHA HELIX CRUISE-173 to western Prince William Sound, Yakutat Bay, and Glacier Bay National Park, northeastern Gulf of Alaska, August 17 - September 3, 1993. 1994, U.S. Geological Survey Open-File Report, 94-258, 94 p.

Edwards, B.D., in press, Variations in sediment texture on the storm-dominated Monterey Bay National Marine Sanctuary continental shelf, Marine Geology, in press.

Gardner, J.V., Edwards, B.D., Dean, W.E. and Wilson, J., 1995, P-wave Velocity, Wet Bulk Density, Magnetic Susceptibility, Acoustic Impedance, and Visual Core Descriptions of Sediment Recovered During Research Cruise EW9504: Data, Techniques, and Procedure. U.S. Geological Survey Open-File Report 95-533, p.3-6.

Kayen, R.E., 1994, Mass Physical and Geotechnical Properties of Sediment on the Palos Verdes Margin: Appendix B to The Distribution and Character of Contaminated Effluent-Effected Sediment, Palos Verdes Margin, Southern California, USGS Administrative Report.

Kayen, R.E. and Phi, T.N., 1997, "A robotics and data acquisition program for manipulation of the U.S. Geological Survey's sediment core logger," SciTech Journal, v. 7, No. 5, 24-29.

Kayen, R.E., Edwards, B.D., and Lee, H.J., 1999. Nondestructive laboratory measurement of geotechnical and geoacoustic properties through intact core-liner, Nondestructive and Automated Testing for Soil and Rock Properties, ASTM STP 1350, W.A. Marr and C.E. Fairhurst, Eds., American Society for Testing and Materials, West Conshohocken, PA, p. 83-94.

Overton, W.S., White, D., and Stevens, D.L., 1990. Design report for EMAP Environmental Monitoring and Assessment Program. U.S. Env. Prot. Agency rpt EPA/600/3-91/053, 52 p.

Stevens, D.L., Jr., and Olsen, A.R., 1991. Statistical issues in environmental monitoring and assessment. Proc. Sec. Statistics and Environ. Am. Statistical Assoc. Alexandria, VA, p. 1-10.

White, D., Kimerling, A.J., and Overton, W.S., 1992. Carographic and geometric components of a global sampling design for environmental monitoring. Cart. And Geog. Info. Sys. v. 9, p. 5-22.

\section{Results}

Spreadsheet of Station Data (416 kb) (table 3)

Index Map (608 kb) (fig. 11)

Surface Texture Map (172 kb) (fig. 6) 
Link back to Sample Collection Procedures chapter

Table 1. Types of subsamples taken and the responsible scientist* .

\begin{tabular}{|l|l|l|l|}
\hline Equipment Code & Sample Type & Responsible Scientist & Organization \\
\hline Surf Tx & Surface $(0-1 \mathrm{~cm})$ Texture sample & Brian D. Edwards & USGS \\
${ }^{14} \mathrm{C}$ and Diatom & ${ }^{14} \mathrm{C}$ dating and Diatom samples & Brian D. Edwards & USGS \\
Surf MF & Surface $(0-1 \mathrm{~cm}$ ) Microfauna sample & Mary L. McGann & USGS \\
SS subcore & Stratigraphy/Sedimentology subcore & Brian D. Edwards & USGS \\
GT subcore & Geotechnical subcore & Brian D. Edwards & USGS \\
GC subcore & Geochemistry subcore & Thomas D. Lorenson & USGS \\
TMSO subcore & Metals, pesticides, \& PCB's subcore & R. Fairey \& M. Stephenson & CDF\&G \\
MB subcore & MacroBenthos subcore & Donald Potts & UCSC \\
MB subcore & MacroBenthos subcore & Amy Wagner & EPA \\
PB subcore & 210Pb subcore & Roger L. Lewis & USGS \\
Bulk sample & Other bulk sediment samples & Brian D. Edwards & USGS \\
Other & Any other samples (e.g., rock samples) & Brian D. Edwards & USGS \\
IGC subcore & Inorganic Geochemistry subcore & Thomas D. Lorenson & USGS \\
OGC subcore & Organic Geochemistry subcore & Keith A. Kvenvolden & USGS \\
MO subcore & Micro-organisms subcore & Bess Ward & UCSC \\
\hline
\end{tabular}

*Inquiries regarding the overall sampling program should be directed to Dr. Brian D. Edwards (bedwards@usgs.gov)

Inquiries regarding disposition of specific subsamples can be directed to the responsible scientist listed in Table 1.

Table 2. Types of subsamples taken from each sampling device.

\begin{tabular}{|c|c|c|c|}
\hline Device & $M-1-95-M B$ & $P-2-95-M B$ & $P-1-97-M B$ \\
\hline \multicolumn{4}{|l|}{ NEL-Box } \\
\hline & \begin{tabular}{|l} 
Surf Tx \\
Surf MF \\
SS subcore \\
GT subcore \\
MF subcore \\
MB Bulk Wash \\
IGC subcore \\
OGC subcore \\
microf subcore \\
Bulk sample \\
Other
\end{tabular} & $\begin{array}{l}\text { Surf Tx } \\
\text { Surf MF } \\
\text { SS subcore } \\
\text { GT subcore } \\
\text { GC subcore } \\
\text { TMSO subcore } \\
\text { MB subcore } \\
\text { MB Bulk Wash } \\
\text { Bulk sample } \\
\text { Other }\end{array}$ & $\begin{array}{l}\text { Surf Tx } \\
\text { Surf MF } \\
\text { SS subcore } \\
\text { GT subcore } \\
\text { GC subcore } \\
\text { TMSO subcor } \\
\text { MB subcore } \\
\text { PB subcore } \\
\text { Bulk sample } \\
\text { Other }\end{array}$ \\
\hline \multicolumn{4}{|c|}{ MultiCorer } \\
\hline & Not Used & $\begin{array}{l}\text { SS subcore } \\
\text { Tx subcore } \\
\text { MF subcore } \\
\text { MB subcore(s) } \\
\text { GT subcore } \\
\text { TMSO subcore }\end{array}$ & Not Used \\
\hline \multicolumn{4}{|c|}{ VanVeen Grab Sampler } \\
\hline & \begin{tabular}{|l} 
Surf Tx \\
Surf MF \\
Bulk sample
\end{tabular} & \begin{tabular}{|l} 
Surf Tx \\
Surf MF \\
Bulk sample
\end{tabular} & \begin{tabular}{|l|} 
Surf Tx \\
Surf MF \\
Bulk sample
\end{tabular} \\
\hline
\end{tabular}

